\title{
Sources of Evidence - The Cochrane Library
}

\author{
Jeyanthi John
}

Senior Researcher, Centre for Evidence-based Density, Oxford UK

\begin{abstract}
This second article in a series on sources of evidence looks at the Cochrane Library. This library contains several electronic databases most notable of which is the Cochrane Database of Systematic Reviews (CDSR) produced by the Cochrane Collaboration. This database is of increasing relevance to dentists thanks to the increasing number of reviews being completed by the members of the Cochrane Oral Health Group.

Evidence-Based Dentistry (2003) 4, 70-72. doi:10.1038/sj.ebd.6400210
\end{abstract}

\section{The Cochrane Collaboration}

The Cochrane Collaboration is an international organisation committed to helping people make well-informed decisions about healthcare by preparing, maintaining and ensuring the accessibility of systematic reviews of healthcare interventions. It is named after Archie Cochrane, a British epidemiologist who recognised the need for high-quality reliable evidence for decision-making in healthcare. The first Cochrane Centre was established in Oxford in the UK in 1992. This has expanded to become the Cochrane Collaboration with Cochrane Centres in 11 countries.

A key function of the Cochrane Collaboration is the production of high-quality systematic reviews, Cochrane Reviews. Collaborative Review Groups, comprising researchers, healthcare professionals, consumers and others, prepare and maintain Cochrane Reviews in various areas of healthcare. Cochrane Methods Groups organise and disseminate good methodology to improve the validity and precision of systematic reviews. Cochrane Fields or Networks are Cochrane groups that focus on aspects of healthcare such as healthcare settings, groups of providers eg, nurses, or types of consumers eg, women. They help Review Groups by searching specialist journals by hand to ensure that the groups' work reflects the priorities and perspectives in their own areas of interest. The Cochrane centres themselves facilitate and co-ordinate the work of the above groups. A
Consumer Network has also been established to actively involve consumers in the work of all the different groups.

\section{The Cochrane Library databases}

The Library is published on CD and on the Internet four times each year, in January, April, July and October. The Internet version can be accessed through the website of the National Library of Health (www.nelh.nhs.uk/cochrane.asp).
The Cochrane Library contains several searchable databases. The first five cover topics relating to evidence-based medicine and the last two provide information on research methodology (Figure 1): The Cochrane Database of Systematic Reviews (CDSR)

This is a database of systematic, up-todate Cochrane reviews of all relevant randomised controlled trials in healthcare. Full-text articles are included of all completed reviews and the protocols of reviews that are still in preparation.

The Database of Abstracts of Reviews of Effectiveness (DARE)

DARE includes structured abstracts of systematic reviews that have been critically appraised by experienced reviewers at the National Health Service Centre for Reviews and Dissemination in the UK.

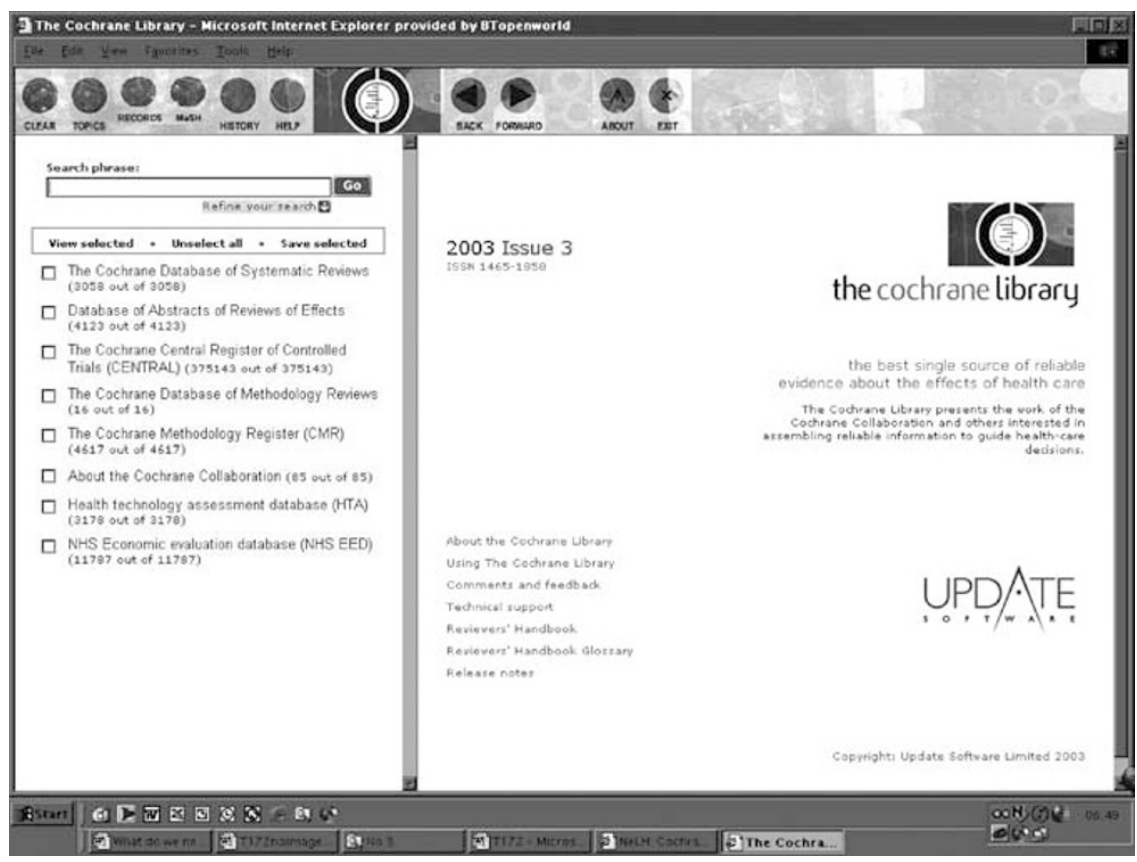

Figure 1. Cochrane Library Index page. 
The Cochrane Central Register of Controlled Trials (CENTRAL)

CENTRAL is a bibliographical register (ie, abstracts only) of all known controlled trials regardless of language of publication or where and when the trial was conducted. The trials are identified from multiple sources including searches of electronic databases, handsearches and searches of other trial registers.

Health Technology Assessment Database (HTA)

This database keeps records of completed and ongoing assessments of the International Network of Agencies of Health Technology Assessments. These assessments investigate the medical, social and ethical implications of health technologies such as pharmaceuticals and surgical procedures.

NHS Economic Evaluation Database (NHS EED)

This contains published economic evaluations of healthcare interventions so that information on costs can be considered alongside effectiveness.

The Cochrane Database of Methodology Reviews

As with the CDSR, this contains complete reviews and protocols of the empirical evidence that underpins the methods used in Cochrane reviews.

The Cochrane Methodology Register (CMR)

CMR is a bibliography of articles on the science of systematic reviews. As with CENTRAL, all known sources of material are searched to identify the maximum number of relevant articles.

\section{Navigating the Cochrane Library}

The website's "Help" sections provide comprehensive explanations about navigating the website and searching the different databases.

Clicking on "Topics" (top left-hand corner of the screen) will give a list of topics, one of which is "Oral Health". This gives access to a list of subjects for which systematic reviews or the protocols for reviews are available.

You can search for specific subjects by typing a term or phrase in the "Search Phrase" box. All databases in the library are searched and the number of 'hits' are listed under each database.

Clicking on a database will produce a drop-down menu listing all the titles produced by the search.
Clicking on a database without entering a search term will produce a dropdown menu, which will enable you to browse through all the titles in it. You can view the full-text of any article by clicking on its title. To print a copy of the article, click on the "Printer-friendly document" icon at the top of the document.

\section{Search tips for the Cochrane Library}

You can search the databases using a variety of methods:

1. Single words or phrases eg, "dentist" will search for all documents that contain the word "dentist", whereas "clinical governance" will search for all documents containing the phrase "clinical governance".

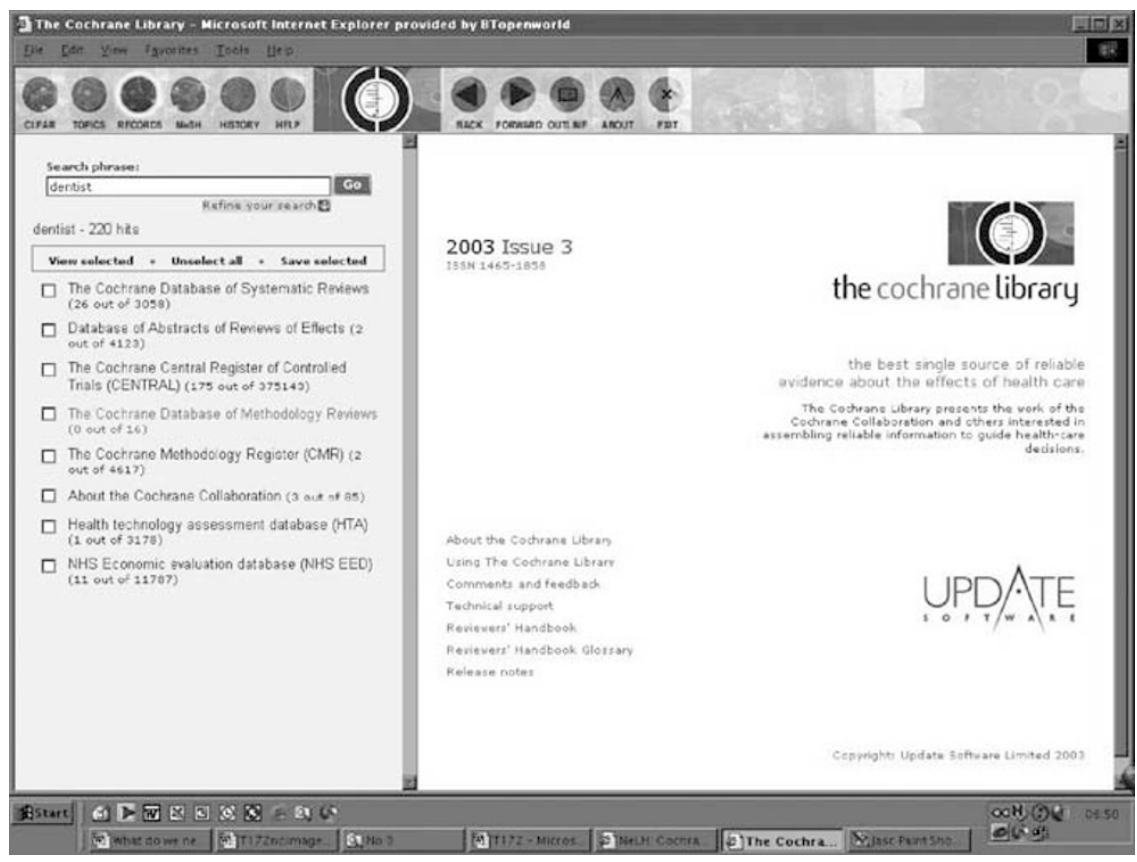

Figure 2. Hits identified using the search term "dentist".

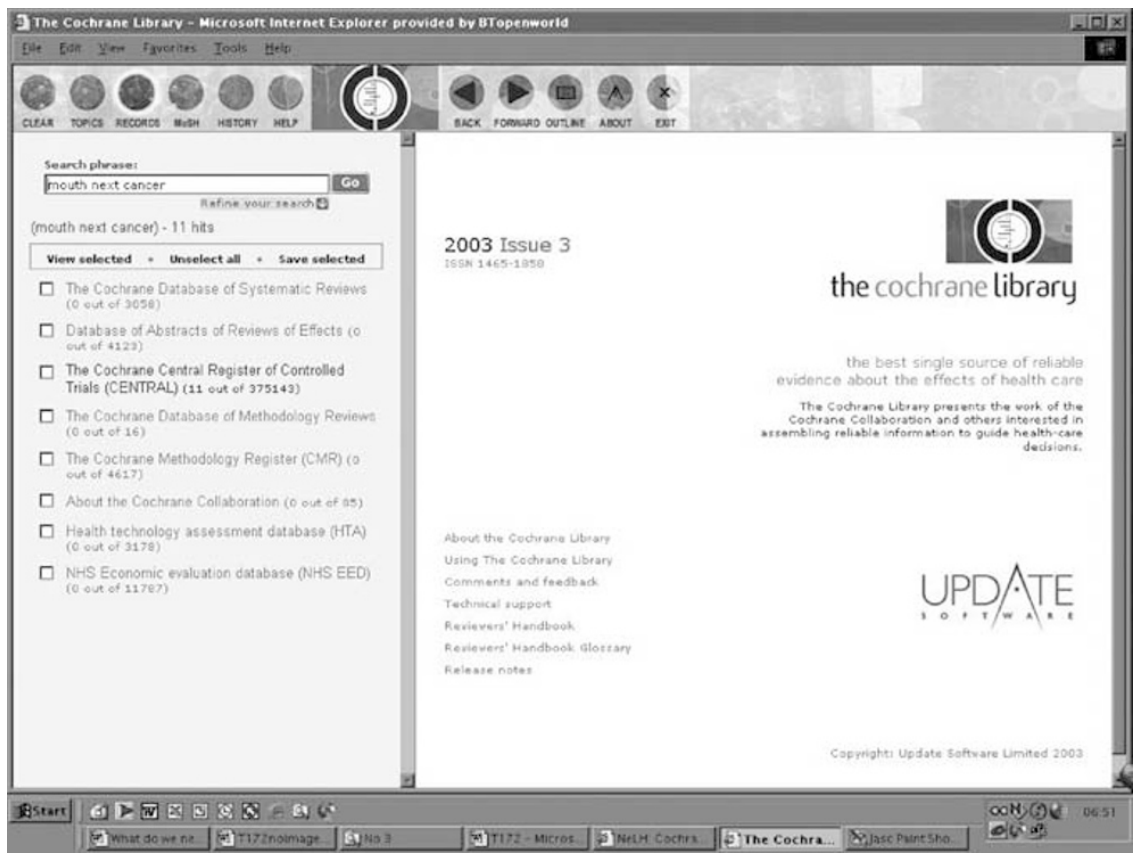

Figure 3. Hits identified using search term "mouth next cancer". 
2. You can combine search terms using Boolean operators such as AND (eg, "dental AND clinic"); OR (eg, "dentist OR dental"); NOT (eg, "mouth NOT foot"); NEXT (eg, "mouth NEXT cancer"); or NEAR (eg, "patch NEAR cancer").

3. Truncation (eg, "dent*") will search for all documents containing words that start with "dent" eg, dentist, dental and dentistry.

You can refine your search by clicking on the "Refine your search" option below the "Search phrase" box. Searches can be restricted to specified date-ranges.
You can also confine your search to look for the search-word (or phrase) in a particular field eg, "smith:au" will retrieve all references listing Smith as the author and "dental:ti" will list all references with the work dental in the title. You can view your search history at any time by clicking on "History" (Figures 2 and 3 ).

\section{Final note}

An important point about Cochrane reviews is that the authors are vested with the responsibility of keeping them up-to-date. An iterative system is in place to ensure that successive versions reflect the relevant new data and valid criticisms from any course. The reader can therefore be confident that the information they are seeing is current. In short, the Cochrane Library is an important and useful source of information for anyone involved in decision-making in healthcare. 